Original Research Paper

\title{
Changes in Prothrombin Time and Platelet Parameters of Mice Treated with Vitamins $\mathbf{C}$ and $\mathbf{E}$
}

\author{
1,2\#Hongwei Li, ${ }^{1 \#}$ Jing Cheng, ${ }^{1 \#}$ Wei Wang, ${ }^{1}$ Xiangcui Chen, \\ ${ }^{1}$ Tongbing Qi, ${ }^{1}$ Xinzhuo Tu, ${ }^{1}$ Jing Zhang, ${ }^{1}$ Weitong Cui and ${ }^{1}$ Qinglu Wang \\ ${ }^{1}$ Key Laboratory of Biomedical Engineering and Technology of Shandong High School, \\ Qilu Medical University, Zibo, 255213, China \\ ${ }^{2}$ Jiangxi Science and Technology Normal University of pharmacy, Jiangxi, 330013, China
}

\author{
Article history \\ Received: 02-01-2020 \\ Revised: 22-02-2020 \\ Accepted: 14-04-2020 \\ Corresponding Authors: \\ Qinglu Wang \\ Key Laboratory of Biomedical \\ Engineering and Technology of \\ Shandong High School, Qilu \\ Medical University, Zibo, \\ 255213, China \\ Email: Wql_zcq@126.com
}

\begin{abstract}
This study aims to elucidate the influence of supplementation with different doses of Vitamin C (VC) and Vitamin E (VE) on Prothrombin Time (PT) in mice; forty eight mice are randomly divided into six treatment groups, which are fed different dose VC and VE except for control Check (CK) for eight weeks. Blood samples are obtained from the mouse canthus to measure PT and platelet parameters. Administration of different doses of VC and VE to mice cause coagulation function damage.
\end{abstract}

Keywords: Vitamin C, Vitamin E, Prothrombin Time, Platelet Parameters

\section{Introduction}

Under normal circumstances, the functions of blood coagulation, anticoagulation, platelet and fibrinolysis in peripheral blood are in a state of dynamic equilibrium, the coagulation mechanism is hyperactive and the anticoagulant mechanism is relatively weakened when this balance is broken. There are many infuence factors of coagulation function, one of which is considered the imbalance of oxidation and anti-oxidation in the body. Under a large number of oxidative free radical condition, endothelial cells injury can cause by inducing oxidative stress injury, endothelial cells release Tissue Factor (TF), TF can trigger endogenous and exogenous coagulation mechanisms Endothelin (ET) and other substances cause vasoconstriction and VW factor causes blood platelet aggregation (Chen, 2005). Acute or chronic liver patients have shown the disorder of coagulation (Imai et al., 2014). Platelet count (PLT), platelet parameters including Mean Platelet Volume (MPV) and platelet hematocrit (PCT) are significantly lower than those of healthy individuals. PT and platelet parameters are used as important clinical reference indicators (Martí-Carvajal and Solà, 2012).

Vitamins are one of the six major nutrients, which can maintain normal metabolism and physiological function (Imai et al., 2014). The clinical applications of vitamins have broadened with the increased understanding of their roles. Vitamin C (VC) is a watersoluble vitamin and a reducing agent. As a scavenger of antioxidant free radicals, it participates in many redox reactions in the human body in clinical practice, $\mathrm{VC}$ is used as a bactericide and antiviral, after Intrauterine Device (IUD) placement, VC can prevent and treat abnormal uterine bleeding and as an adjuvant therapy for viral myocarditis (Martí-Carvajal and Solà, 2012; Guo, 2014). High doses of VC can be safely used to treat diseases. Patients with Immune Thrombocytopenia (ITP) treat with high-dose ( $2 \mathrm{~g} /$ day) vitamin $\mathrm{C}$ and their platelet count reach $50 * 10^{9} / \mathrm{L}$ after 7 days. And then other clinical treatment centers use vitamin $\mathrm{C}$ to treat ITP patients also achieved good results (Meng, 2017). The mechanism of action of vitamin $\mathrm{C}$ in ITP may be related to the change of $\mathrm{pH}$ in the circulatory system, which reducing the capacity of the mononuclearmacrophage system and reducing the destruction of platelets. Vitamin $\mathrm{C}$ also promotes the synthesis of intercellular substance in connective tissue, inhibites fibrinolysis and improves cell integrity, reduces capillary permeability and platelet physical injury (Sasidharan Nair et al., 2016; Huijskens et al., 2014).

Vitamin E (VE) is a fat-soluble vitamin and an important antioxidant. It can maintain the integrity of tissue structure, promote fertility, protect against cancer, support a healthy cardiovascular system and treat some diseases of premature infants (Wang et al., 2014). VE combines with low molecular weight heparin sodium which prolonged clotting time and less caused a decrease in the number of platelets in Preeclampsia (Riordan et al., 2000). 
The number of platelet in immune thrombocytopenia patients increase by using large dose of VC (Meng, 2017). Administration VE and low molecular weight heparin sodium prolong clotting time and barely decrease the number of platelet in Preeclampsia. VE and VC, two important antioxidant vitamins in the body, have synergistic effects (Xun and Mei, 2004; Xiao and $\mathrm{Li}, 2015)$. The combination of these two vitamins with VK can affect VK function. PT and platelet parameters of patients with hepatic cirrhosis directly respond the therapeutic effect of vitamin K (Ijland et al., 2008; Zong, 2008). VC, VE and their combination have protective effect on vascular endothelial cell following oxidative injury in vitro (Manoharan, 1991). However, in wang' s study, wistar rats were randomly divided into six groups including control, VC (1000 mg/kg VC), VE1 (33 mg/kg $\mathrm{VE}), \mathrm{VE} 2(500 \mathrm{mg} / \mathrm{kg} \mathrm{VE}), \mathrm{VC}+\mathrm{VE} 1(1000 \mathrm{mg} / \mathrm{kg} \mathrm{VC}$ and $33 \mathrm{mg} / \mathrm{kg} \mathrm{VE}), \mathrm{VC}+\mathrm{VE} 2(1000 \mathrm{mg} / \mathrm{kg} \mathrm{VC}$ and 500 $\mathrm{mg} / \mathrm{kg} \mathrm{VE})$. Relatively high dose VE could effectively enhance lymphocyte proliferation, DNA stability and erythrocyte membrane fluidity. But high dose VC could cause antagonism effect. Excessive VE and VC show no favourable effects and the activities of peripheral blood cell might be decreased (Wang et al., 2006). Platelet count and morphology and vascular endothelial cells are related with coagulation, VC, VE and their combination have different effects on these aspects, but most studies are in vitro or hypercoagulable. prothrombin time and platelet parameters directly show the coagulation function and platelet morphology and the detection method is very mature.

In this study, we designed two experiments to expound the chang of Prothrombin Time (PT), Platelet count (PLT), Platelet hematocrit (PCT), platelet distribution width (PDW) and Mean Platelet Volume (MPV) of mice, which were treated with vitamins $\mathrm{C}$ and $\mathrm{E}$.

\section{Materials and Methods}

\section{Animals}

Forty eight healthy four-week-old mice were provided by Beijing Lihua Experimental Animal Technology Co. Ltd., Beijing (Certificate number: Scxk). Half male and female, at room temperature (approximately $20^{\circ} \mathrm{C}$ ) and $60 \%$ relative humidity with a $12 \mathrm{~h}$ light/dark cycle. eight mice were reared per cage and provided food and water ad libitum. The mice were allowed to adapt to the experimental environment for one week. All animal procedures were approved by the Qilu Medical University Animal Investigational Committee and were performed in accordance with the Guide for the Care and Use of Laboratory Animals published by the Ministry of Health of People's Republic of China and IJSM's ethical standards document, this study has been approved by the ethics committee of Qilu Medical University.

\section{Chemicals and Reagents}

Effervescent VC tablets were purchased from Bayer HealthCare AG. Soft VE capsules were purchased from Zhejiang Pharmaceutical Limited Share Ltd. A commercial PT kit was purchased from Wuhan Zhongtai Biotechnology Co. Ltd. XT-1800i blood analyzer was purchased from Sysmex Company.

\section{Experimental Groups}

Mice were allowed to adapt to the experimental environment for one week and were then randomly divided into six groups, as follows: $\mathrm{CK}$ - blank control (saline); VC- VC control (1000 mg/kg VC); VEL- low dose of VE (33 mg/kg VE); VEH- high dose of VE group (VEH); CLE- high dose of VC combined with a low dose of VE (1000 mg/kg VC and $33 \mathrm{mg} / \mathrm{kg} \mathrm{VE})$; and CHE- high dose of VC combined with a high dose of VE $(1000 \mathrm{mg} / \mathrm{kg} \mathrm{VC}$ and $500 \mathrm{mg} / \mathrm{kg} \mathrm{VE})$. Each group comprised four mice.

\section{Treatment Administration and Blood Sample Collection}

Treatment was administered in accordance with mouse weight. Drugs were chronically administered to animals for 8 weeks. Blood samples were taken from the eye canthus of each mouse after the last episode of drug administration (with anesthesia). Blood was collected in $2 \mathrm{~mL}$ EDTA- $\mathrm{K}_{2}$ blood vials. The vials were shaken to mix blood samples prior to the detection of platelet parameters. Blood samples were then transferred into EP tubes containing $0.109 \mathrm{~mol} / \mathrm{L}$ sodium citrate as an anticoagulant. The volume ratio of blood samples to sodium citrate is 9:1. The vials were shaken and centrifuged for 15 minutes at $3000 \mathrm{rpm}$. The supernatant, which contained platelet-poor plasma, was transferred to new EP tubes and stored at $-20^{\circ} \mathrm{C}$ until use.

\section{Determination of PT}

PT reagent was added to $1 \mathrm{ml}$ complex solution. The mixture was gently mixed and allowed to stand for 15 min at room temperature. An appropriate amount of PT was collected, incubated at $37^{\circ} \mathrm{C}$ and used in accordance with the instructions presented in Table 1.

Table 1: Test Methods of prothrombin time

\begin{tabular}{lc}
\hline Added substance & Volume \\
\hline Plasma & $100 \mu \mathrm{L}$ \\
$\begin{array}{l}\text { Incubation for } 3 \text { minutes at } 37{ }^{\circ} \mathrm{C} \\
\text { Prothrombin time reagent }\end{array}$ & $200 \mu \mathrm{L}$ \\
\hline $\begin{array}{l}\text { Immediately mix the sample and record the time of clotting } \\
\text { (repeat twice) }\end{array}$
\end{tabular}




\section{Determination of Platelet Parameters}

The PLT, PDW, MPV and PCT of blood samples were analyzed by XT-1800i blood analyzer. For the manual test, $85 \mu \mathrm{L}$ of blood sample was used. First, the manual key on the toolbar was selected and a sample ID number was set. The option "Analyze project" was selected. Then, the "Manual analysis mode" was selected. The test results were then obtained.

\section{Statistical Analysis}

All data were analyzed using SPSS13.0 statistical software. Results were expressed as mean and standard deviation $(\bar{x} \pm s)$. For the comparison of two groups, Pvalues were calculated by two-tailed unpaired Student's $t$-test. Comparison between groups was conducted using single-factor analysis of variance. $\mathrm{P}<0.05$ indicated significance and $\mathrm{P}<0.01$ indicated extreme significance.

\section{Results}

\section{General Conditions of Laboratory Animals}

The weights of the mice increased normally during the experiment. Mice in the VEH and VC\&VEH groups exhibited bloody stool. The humane endpionts were that mice' hair turned yellow and messy and no luster and they showed sleepiness, mental apathetic state (Sun et al., 2014; Miller et al., 2005).

The homogeneity of variance of PT, PLT and PCT are shown in Table 2. The results presented in Table 2 indicated that PT first shortened and then lengthened. The PT of the VC control group was shorter than that of the $\mathrm{CK}$ group. By contrast, the PT of the $\mathrm{CEH}$ group was longer than those of the VEL, VEH and CLE groups. PLT first increased and then decreased. Among all groups, the VC control group had the highest PLT. The PLT of the VEL, VEH, CLE and CHE groups decreased. PCT first increased and then decreased and the VC control group had the highest PCT among all groups. The PCT of the VEL, VEH, CLE and CHE groups decreased. $F$-test between the PDW and MPV of different groups revealed an P-value of $0.738,0.740$ $(\mathrm{p}>0.05)$, indicating no significant difference.

\section{Multiple Comparison of Prothrombin Time (PT)}

Prothrombin time was measured in accordance with the instructions included with the PT kit. Results showed in Fig. 1 and Table 2. PT in CHE group significantly $(\mathrm{P}<0.05)$ higher than $\mathrm{CK}$ group. But there was no significant difference in prothrombin time between the other treatment groups and the CK group $(\mathrm{P}>0.05)$. Specifically, VC, VEL, VEH, CLE and CHE group compared with each other, the prothrombin time of the VC group was significantly shorter than that of the VEH, CLE and CHE group $(\mathrm{P}<0.05)$, whiling PT of the $\mathrm{CEH}$ group was significantly longer than that of the CLE group $(\mathrm{P}<0.05)$.

\section{Multiple Comparison of Platelet Parameters}

Results are shown in Fig. 2 and Table 2. Platelet count (PLT) first increased and then decreased. PLT significantly differed among $\mathrm{CK}$ and the administration groups $(\mathrm{P}<0.05)$. Meanwhile comparison of $\mathrm{VC}$, VE group and their combination groups, PLT in VC group significantly increased when compared with VEL, VEH, CLE, CHE groups $(\mathrm{P}<0.05)$ and the result showed that PLT gradually decreased in VEL, VEH, CLE, CHE groups. Nevertheless, PLT in low dose of VC, VE combined group with high dose of $\mathrm{VC}, \mathrm{VE}$ combined group $(\mathrm{P}>0.05)$.

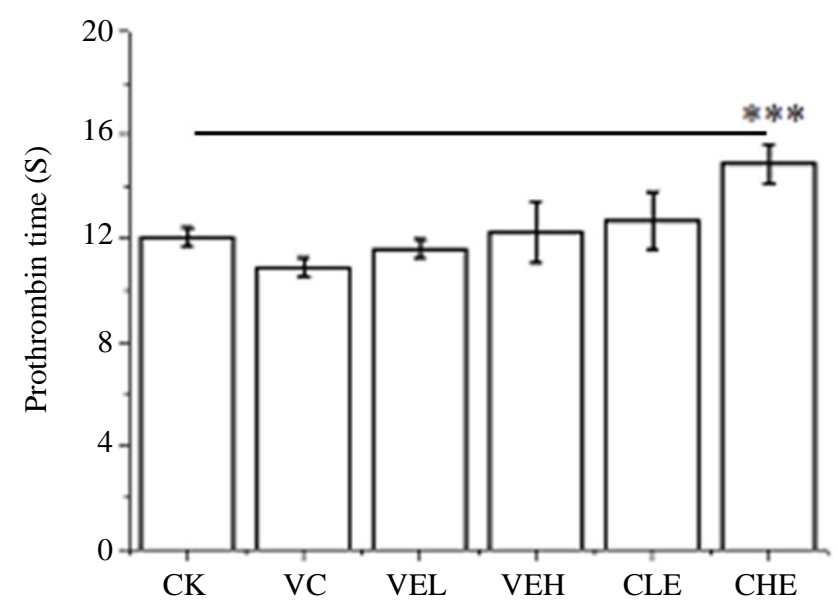

Fig. 1: The effect of VC, VE and their combinations on prothrombin time (PT) of mice; *** The mean difference is significant at the 0.001 level ( $\mathrm{P}<0.001)$, compared with control group. CK- blank control (saline); VC- VC control (1000 mg/kg VC); VELlow dose of VE (33 mg/kg VE); VEH- high dose of VE group (VEH); CLE- high dose of VC combined with a low dose of VE (1000 mg/kg VC and $33 \mathrm{mg} / \mathrm{kg} \mathrm{VE})$; and CHE-high dose of VC combined with a high dose of VE (1000 mg/kg VC and $500 \mathrm{mg} / \mathrm{kg} \mathrm{VE})$. 


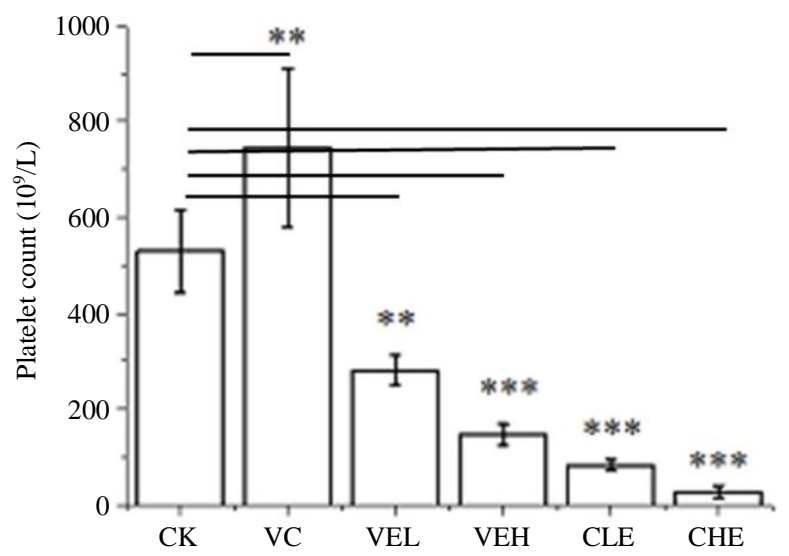

Fig. 2: The effect of VC, VE and their combinations on Platelet Count (PCT) of mice; $* *$ The mean difference is significant at the 0.01 level $(\mathrm{P}<0.01)$, *** The mean difference is significant at the 0.001 level $(\mathrm{P}<0.001)$. compared with control groupCKblank control (saline); VC- VC control (1000 mg/kg VC); VEL- low dose of VE (33 mg/kg VE); VEH- high dose of VE group (VEH); CLE- high dose of VC combined with a low dose of VE (1000 mg/kg VC and $33 \mathrm{mg} / \mathrm{kg} \mathrm{VE})$; and CHE high dose of VC combined with a high dose of VE (1000 mg/kg VC and $500 \mathrm{mg} / \mathrm{kg} \mathrm{VE})$.

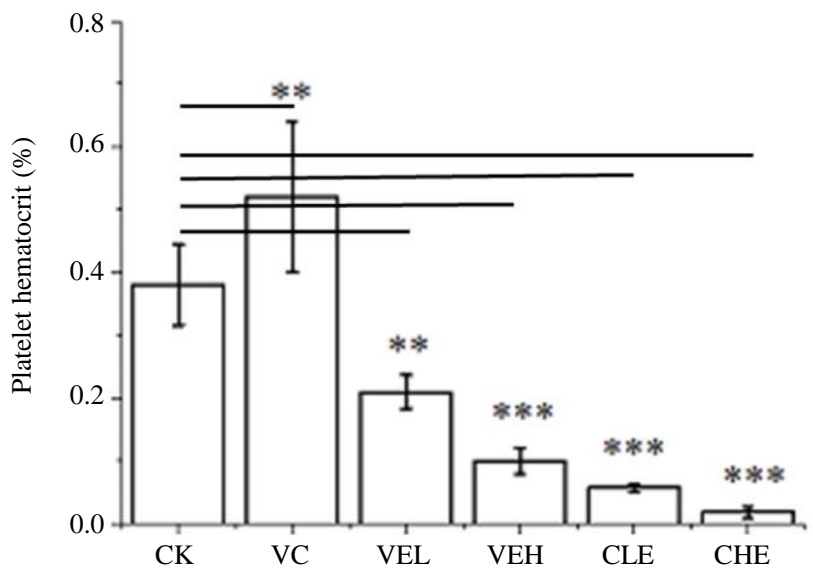

Fig. 3: The effect of VC, VE and their combinations on platelet hematocrit (PCT) of mice; ** The mean difference is significant at the 0.01 level $(\mathrm{P}<0.01)$, *** The mean difference is significant at the 0.001 level $(\mathrm{P}<0.001)$. compared with control group.CK- blank control (saline); VC- VC control (1000 mg/kg VC); VEL- low dose of VE (33 mg/kg VE); VEH- high dose of VE group (VEH); CLE- high dose of VC combined with a low dose of VE (1000 mg/kg VC and $33 \mathrm{mg} / \mathrm{kg} \mathrm{VE})$; and CHE high dose of VC combined with a high dose of VE (1000 mg/kg VC and $500 \mathrm{mg} / \mathrm{kg} \mathrm{VE})$

Table 2: Means $(\bar{x} \pm s)$ and homogeneity test of variance results of PT, PLT, PDW, MPV and PCT

\begin{tabular}{lllllll}
\hline Group & $\mathrm{n}$ & PT $(\mathrm{s})$ & PLT $\left(10^{\wedge} 9 / \mathrm{L}\right)$ & PDW (fL) & MPV (fL) & PCT (\%) \\
\hline CK & 4 & $12.05 \pm 0.35$ & $529 \pm 87.07$ & $7.40 \pm 0.17$ & $7.10 \pm 0.064$ & $0.38 \pm 0.064$ \\
VC & 4 & $10.89 \pm 0.37$ & $744 \pm 164.85^{\mathrm{a}}$ & $7.52 \pm 0.43$ & $6.98 \pm 0.12$ & $0.52 \pm 0.12^{\mathrm{a}}$ \\
VEL & 4 & $11.60 \pm 0.34$ & $282 \pm 30.78^{\mathrm{ab}}$ & $7.82 \pm 0.3$ & $7.32 \pm 0.026$ & $0.21 \pm 0.026^{\mathrm{ab}}$ \\
VEH & 4 & $12.23 \pm 1.18^{\mathrm{b}}$ & $146.67 \pm 22.19^{\mathrm{abc}}$ & $7.37 \pm 0.12$ & $6.97 \pm 0.021$ & $0.10 \pm 0.021^{\mathrm{abc}}$ \\
CLE & 4 & $12.70 \pm 1.12^{\mathrm{bd}}$ & $82.7 \pm 10.97^{\mathrm{abc}}$ & $6.93 \pm 0.058$ & $7.13 \pm 0.0058$ & $0.057 \pm 0.0058^{\mathrm{abc}}$ \\
CHE & 4 & $14.87 \pm 0.74^{\mathrm{ab}}$ & $27.25 \pm 13.94^{\mathrm{abc}}$ & $7.12 \pm 1.26$ & $7.00 \pm 0.59$ & $0.018 \pm 0.0096^{\mathrm{abc}}$ \\
F & & 14.026 & 43.222 & 0.547 & 0.545 & 39.840 \\
P & & 0.000 & 0.000 & 0.738 & 0.740 & 0.000 \\
\hline
\end{tabular}

a: $\mathrm{P}<0.05$ vs CK group; b: $\mathrm{P}<0.05$ Vs VC group; c: $\mathrm{P}<0.05$ Vs VEL group; $\mathrm{d} \mathrm{P}<0.05$ Vs CHE. CK- blank control (saline); VC-VC control (1000 mg/kg VC); VEL- low dose of VE (33 mg/kg VE); VEH- high dose of VE group (VEH); CLE- high dose of VC combined with a low dose of VE (1000 mg/kg VC and $33 \mathrm{mg} / \mathrm{kg} \mathrm{VE})$; and CHE high dose of VC combined with a high dose of VE $(1000 \mathrm{mg} / \mathrm{kg} \mathrm{VC}$ and $500 \mathrm{mg} / \mathrm{kg} \mathrm{VE})$ 
The effect of VC, VE and their combined effect on Platelet hematocrit (PCT) of mice are shown in Fig. 3 and Table 2.

PCT ratio first increased and then decreased. The PCT ratio of the CK group is significantly $(\mathrm{P}<0.05)$ different from that of other groups. The result suggested that Using VC or VE alone has an effect on PT. Comparing VC and CK groups, PT in VC group significantly increased $(\mathrm{P}<0.05)$, then $\mathrm{PT}$ in $\mathrm{VEL}$, VEH. group significantly decreased when compared with VC groups. Comparing the effect of VC, VE and their combinations on Platelet hematocrit (PCT) of mice. PLT significantly decreased in CLE, CHE groups when compared with VEL group, however there is no significantly different between CLE and CHE groups.

\section{Multiple Comparative Analysis of PDW and MPV}

The results are shown in Table 2 and in Fig. 4 and 5.

F-test between the PDW and MPV of different groups revealed an $\mathrm{p}$-value of $0.738,0.740(\mathrm{p}>0.05)$, indicating no difference. $\mathrm{VC}, \mathrm{VE}$ and their combinations have no effect on the platelet distribution width (PDW) and mean platelet volume (MPV) of mice.

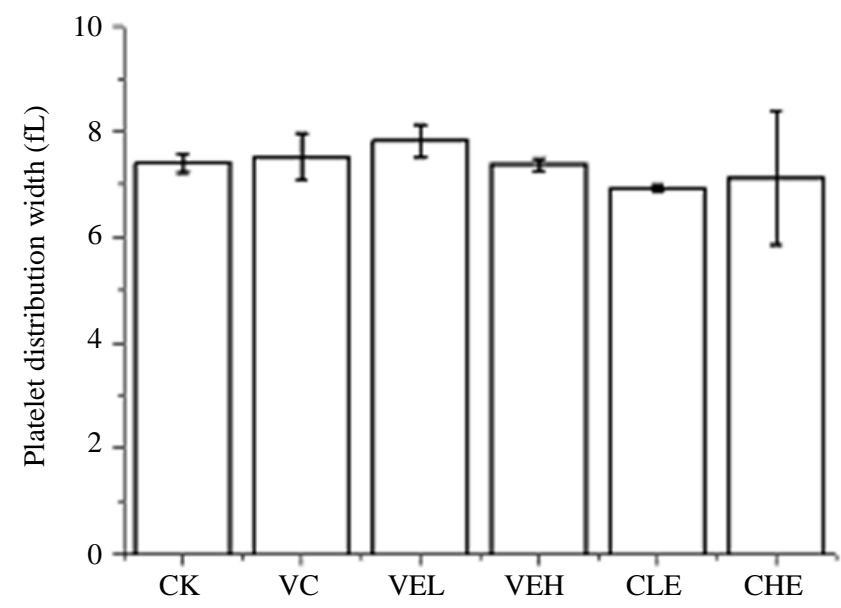

Fig. 4: The effect of VC, VE and their combinations on platelet distribution width (PDW) of mice; CK- blank control (saline); VCVC control (1000 mg/kg VC); VEL- low dose of VE (33 mg/kg VE); VEH- high dose of VE group (VEH); CLE- high dose of VC combined with a low dose of VE (1000 mg/kg VC and $33 \mathrm{mg} / \mathrm{kg} \mathrm{VE})$; and CHE high dose of VC combined with a high dose of VE (1000 mg/kg VC and $500 \mathrm{mg} / \mathrm{kg} \mathrm{VE})$.

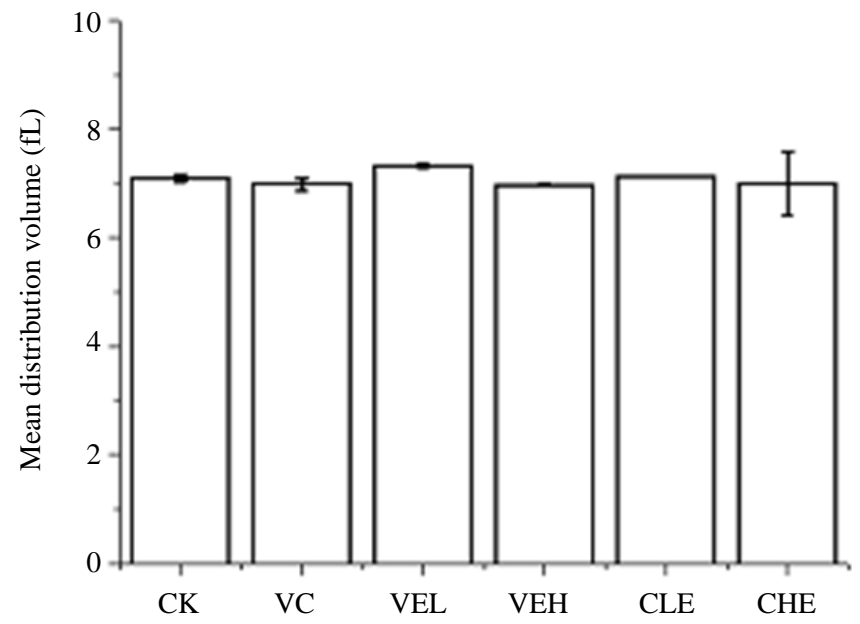

Fig. 5: The effect of VC, VE and their combinations on mean platelet volume (MPV) of mice; CK- blank control (saline); VC- VC control (1000 mg/kg VC); VEL- low dose of VE (33 mg/kg VE); VEH- high dose of VE group (VEH); CLE- high dose of VC combined with a low dose of VE (1000 mg/kg VC and $33 \mathrm{mg} / \mathrm{kg} \mathrm{VE})$; and CHE high dose of VC combined with a high dose of VE $(1000 \mathrm{mg} / \mathrm{kg} \mathrm{VC}$ and $500 \mathrm{mg} / \mathrm{kg} \mathrm{VE})$ 


\section{Discussion}

In this study, mouse was used as experimental model for the analyzing effect of high-dose vitamin on clotting mechanism. It is well known that human and guinea pig are L-gulonolactone oxidase deficient, however mouse is able to metabolize glucose to vitamin C. So mouse model may not be used in lowdose vitamin $\mathrm{C}$ analysis. study about high-dose vitamin $\mathrm{C}$ in mouse (Kondo et al., 2019).

In Xie's study, The amount of gastric lavage was calculated according to the body weight of mice $(0.01$ $\mathrm{ml} / \mathrm{g}$ ), the bleeding time in the VE high concentration group $(5.0 \mathrm{mg} / \mathrm{ml})$ and the heparin group was longer than that in the normal control group and the etamsylate group $(P<0.01)$. VE has no effect on blood coagulation and erythrocyte membrane stability, it can prolong physiological hemosta-sis time and there is dose effect relationship in this effect. In this study the prothrombin time increased, Platelet count (PLT) and Platelet hematocrit (PCT) decreased. In wang's study (Wang et al., 2006), high dose VE (500 mg/kg) could effectively enhance lymphocyte proliferation, DNA stability and erythrocyte membrane fluidity.. excessive VE and VC showed no favourable effects and the activities of peripheral blood cell might be decreased. In this study, high dose VC and high dose VE have synergistic effects on coagulation mechanisms.

PT and activated partial PT are the most widely used parameters in the investigation of coagulation abnormalities. However, the interpretation of these parameters has been rather confusing given their nonstandardized descriptions in clinical settings. PT results have been reported as clotting time, percentage activity, patient-to-normal clotting time, or International Normalized Ratio (INR). The INR scale has been devised to standardize results stemming from different thromboplastins from patients under treatment with VK antagonists (Mi et al., 2017).

VE can extend physiological bleeding time in a dosedependent manner. In this study, VE treatment extended the physiological bleeding time. This effect may be linked to vasoconstriction and PT (Guo et al., 2017). VE can inhibit platelet aggregation and prevent thrombosis given that it affects the synthesis of platelet prostacyclin and impairs platelet aggregation by disrupting PGI2/TXA2 balance (Vucinic et al., 2010). After taking vitamin $\mathrm{E}$, patients with hyperlipidemia and fatty liver show blood in the stool and sputum, patients stop taking VE and taking lutin tablets three times a day, the stool turns yellow and the bleeding point of the right eye disappears. Infantile immune thrombocytopenic purpura has been effectively treated with high doses of VC and gluocoritocoids (Shi, 2012). In this study, combination therapy with high doses of VC improved symptoms and increased PLT. High doses of VC may have induced the mononuclear macrophage system to promote platelet production by promoting bone marrow megakaryocyte differentiation and maturation.

In this study, PT time first shortened and then lengthened and PLT first increased and then decreased (Fig. 1). PCT ratio first increased and then decreased (Fig. 2). The PT of the VC control group was shorter than that of the other groups $(\mathrm{P}<0.05)$. The $\mathrm{VC}$ group had higher platelet counts than the control, VEL, VEH and CLE groups. PCT ratio increased in the VC group. Thus, treatment with high doses of VC increased PLT and PCT ratio but decreased PT. However, treatment with high doses of VE decreased PLT and PCT ratio but increased PT in the VEL and VEH groups compared with that in the CLE and CHE groups. VE treatment may have affected the synthesis of platelet prostacyclin and disrupted PGI2/TXA2 balance, thus leading to platelet aggregation. Therefore, the combined use of VC and VE decreased coagulation function in mice. The limitations of this study this experiment only used mice, We should also do further research with other animals, such as rats and rabbits. $\alpha$-tocopherol and $\beta$-tocopherol can inhit lipid peroxidation, erythrocyte hemolysis, liver necrosis (Mah et al., 2013; Devaraj et al., 2008). After taking $\alpha$-tocopherol and $\beta$-tocopherol, compares the influence factors of thrombus and platelet aggregation in rats, $\beta$-tocopherol can be more effective in reducing platelet aggregation and delaying arterial thrombosis (Saldeen et al., 1999). $\alpha$-tocopherol and $\beta$-tocopherol improves blood clotting and platelet aggregation caused by exercise (Vucinic et al., 2010).

\section{Acknowledgement}

This work was supported by grants from the Natural Scientific Foundation of Shandong Province, China (ZR2018MH038), the National Natural Scientific Foundation of China (31701042) and the Technology Development Project Plan of Shandong Education Department (J17KA102), Zibo Platform for Gene Editing and Cell Application (2018ZBXC010, 2018ZBXC008).

\section{Authors Contributions}

Hongwei Li, Jing Cheng and Wei Wang: Participated in all experiments, coordinated the dataanalysis and contributed to the writing of the manuscript.

Xiangcui Chen, Tongbing Qi and Xinzhuo Tu: Coordinated the mouse work.

Jing Zhang and Weitong Cui: Participated in partial experiments.

Qinglu Wang: Designed the research plan and organized the study.

\section{Conflict of Interest}

The authors declare that there are no conflicts of interest. 


\section{Disclosure}

The authors declare that there are no conflicts of interest in this work.

\section{Reference}

Chen, Z.C., 2005. Pathophysiology. People's Health Publishing House.

Guo, J.P., 2014. Reasonable application of vitamins and precautions. North Pharmacy, 11: 99-99.

Guo, Y.X., L. Ming and F.F. Wen, 2017. Effects of vitamin $\mathrm{E}$ on bleeding time, clotting time and red cell osmotic fragility in mice. Chinese Youjiang Med. J., 4: 459-461.

Huijskens, M.J., M. Walczak, N. Koller, J.J. Briedé and B.L. Senden-Gijsbers et al., 2014. Technical advance: Ascorbic acid induces development of double-positive $\mathrm{T}$ cells from human hematopoietic stem cells in the absence of stromal cells. J. Leukoc Biol., 96: 1165-75. DOI: 10.1189/jlb.1TA0214-121RR

Imai, K., K. Takai, T. Hanai, M. Shiraki and Y. Suzuki et al., 2014. Impact of serum chemerin levels on liver functional reserves and platelet counts in patients with hepatocellular carcinoma. Int. J. Mol. Sci., 15: 11294-306. DOI: 10.3390/ijms 150711294

Ijland, M., R. Pereira and E. Cornelissen, 2008. Incidence of late vitamin $\mathrm{k}$ deficiency bleeding in newborns in the Netherlands in 2005: Evaluation of the current guideline. Eur. J Pediatr., 167: 165-169. DOI: 10.1007/s00431-007-0443-X

Kondo, K., K. Hiramoto, Y. Yamate, K. Goto and H. Sekijima et al., 2019. Ameliorative effect of highdose vitamin $\mathrm{C}$ administration on dextran sulfate sodium-induced colitis mouse model. Biol. Pharm. Bull., 42: 954-959. DOI: 10.1248/bpb.b18-00967

Martí-Carvajal, A. and I. Solà, 2012. vitamin K for upper gastrointestinal bleeding in patients with acute or chronic liver disease. Cochrane Database Systematic Rev. DOI: 10.1002/14651858.CD006007.pub3

Meng, W., 2017. Conventional treatment with high doses of vitamin $\mathrm{C}$ therapy pediatric clinical analysis of myocarditis. Clin. Med., 4: 208-209.

Miller, E.R., R. Pastor-Barriuso, D. Dalal, R.A. Riemersma and L.J. Appel et al., 2005. High-dosage vitamin E supplementationmay. Ann. Intern. Med., 142: 37-46.

DOI: 10.7326/0003-4819-142-1-200501040-00110

Mi, Y.N., N.N. Ping, B. Li, X. Xiao and Y.B. Zhu et al., 2017. Finding the optimal dose of vitamin K1 to treat vitamin $\mathrm{K}$ deficiency and to avoid anaphylactoid reactions. Fundam. Clin. Pharmacol., 31: 495-505. DOI: 10.1111/fcp. 12290

Riordan, N., H. Riordan and J. Casciari, 2000. Clinical and experimental experiences with intravenous vitamin C. J. Orthomolecular Med., 15: 201-213.
Sasidharan Nair, V., M.H. Song and K.I. Oh, 2016. Vitamin C facilitates demethylation of the foxp3 enhancer in a tet-dependent manner. J. Immunol., 196: 2119-2131. DOI: 10.4049/jimmunol.1502352

Sun, J.C., Q. Wei, Y.T. Wu, Z.X., Ren and S.P. Ren et al., 2014. Effects of high dose vatimin $\mathrm{E}$ on oxidative stress and thyroid function of rats. J. Jilin Univ., 40: 281-284.

Shi, Y.L., 2012. Efficacy of high-dose vitamin C and glucocorticoid on infantile immune thrombocytopenic purpura. China Modern Med., 19: 238.

Wang, X.L., J.L. Zhang and W.N. Guo, 2014. Vitamin E, low molecular weight heparin combined with magnesium sulfate in the treatment of early onset preeclampsia. Hebei Med. J., 36: 1963-1964.

Wang, Q.Z., A.G. Ma and Y.Y. Sun, 2006. Effects of high dose VE and VC on cell activities in rats. Chin, J. Public Health, 22: 9.

Xun, X.M. and Q.C. Mei, 2004. Clinical research on safety of fat soluble vitamins. Chinese J. Clin. Nutrit., 12: 280-283.

Xiao, H.L. and Z.L. Li, 2015. Clinical observation of 106 cases of abnormal uterine bleeding caused by intrauterine device combined with tranexamic acid and vitamin E. Mod. Diagn. Treat., 26: 2968-2969.

Zong, J.M., 2008. Interaction between vitamins. J. Pharmacist, 2: 24-25.

Vucinic, L., I. Singh, F.J. Spargo, J.A. Hawley and M.D. Linden, 2015. Gamma tocopherol supplementation prevents exercise induced coagulation and platelet aggregation[J]. Thromb. Res., 125: 196-199. DOI: 10.1016/j.thromres.2009.11.015

Mah, E., S.K. Noh, K.D. Ballard, H.J. Park and J.S. Volek et al., 2013. Supplementation of a gammatocopherol-rich mixture of tocopherols in healthy men protects against vascular endothelial dysfunction induced by postprandial hyperglycemia[J]. J. Nutr. Biochem., 24: 196-203.

DOI: $10.1016 /$ j.jnutbio.2012.04.015

Manoharan, A., 1991. Treatment of refractory idiopathic thrombocytopenic purpura in adults. Brit. J. Haematol., 79: 143-147.

Devaraj, S., S. Leonard, M.G. Traber and I. Jialal, 2008. Gamma-tocopherol supplementation alone and in combination with alpha-tocopherol alters biomarkers of oxidative stress and inflammation in subjects with metabolic syndrome[J]. Free Radic. Biol. Med., 44: 1203-1208.

DOI: $10.1016 /$ j.freeradbiomed.2007.12.018

Saldeen, T., D. Li and J.L. Mehta, 1999. Differential effects of alpha- and gamma-tocopherol on lowdensity lipoprotein oxidation, superoxide activity, platelet aggregation and arterial thrombogenesis[J]. J Am. Coll. Cardiol., 34: 1208-15. DOI: 10.1016/S0735-1097(99)00333-2 